\title{
In vivo CT imaging of gold nanoparticle-labeled exosomes in a myocardial infarction mouse model
}

\author{
Lianggeng Gong ${ }^{1}$, Yingying Weng ${ }^{1}$, Wei Zhou ${ }^{1}$, Kunchi Zhang ${ }^{2}$, Wei Li ${ }^{2}$, Jia Jiang ${ }^{3}$, Jun Zhu ${ }^{4}$ \\ ${ }^{1}$ Department of Radiology, the Second Affiliated Hospital of Nanchang University, Nanchang, China; ${ }^{2}$ Shanghai Key Laboratory of Molecular \\ Imaging, Shanghai University of Medicine \& Health Sciences, Shanghai, China; ${ }^{3}$ Department of Sports Medicine, Shanghai Jiaotong University \\ Affiliated Sixth People's Hospital, Shanghai, China; ${ }^{4}$ Research Laboratory for Functional Nanomaterial, National Engineering Research Center for \\ Nanotechnology, Shanghai, China \\ Contributions: (I) Conception and design: L Gong, W Li; (II) Administrative support: J Jiang, J Zhu; (III) Provision of study materials or patients: L \\ Gong; (IV) Collection and assembly of data: Y Weng, W Zhou, K Zhang; (V) Data analysis and interpretation: L Gong; (VI) Manuscript writing: All \\ authors; (VII) Final approval of manuscript: All authors. \\ Correspondence to: Wei Li. Shanghai Key Laboratory of Molecular Imaging, Shanghai University of Medicine \& Health Sciences, Shanghai, China. \\ Email: liwei860130@gmail.com; Jia Jiang. Department of Sports Medicine, Shanghai Jiaotong University Affiliated Sixth People’s Hospital, \\ Shanghai, China. Email: jessicajj19@hotmail.com; Jun Zhu. National Engineering Research Center for Nanotechnology, Shanghai, China. \\ Email: yzjzhu@alumni.sjtu.edu.cn.
}

Background: Acute myocardial infarction (MI) is the primary factor leading to cardiovascular diseases, which are the main causes of morbidity and mortality in developed countries. Mesenchymal stem cell (MSC)-derived exosomes have been reported to improve heart function after MI; however, the molecular mechanisms responsible for this are unknown. In vivo imaging can reveal the trafficking process and in vivo biodistribution of exosomes, which may provide an insight into the communication mechanisms and pharmacokinetics of exosomes.

Methods: Glucose modified gold nanoparticles were used to label MSC-derived exosomes, aimed at minimizing membrane damage and maintaining the integrity of the exosomes. After labeling, the exosomes were visualized by in vivo computed tomography (CT) imaging to determine the biodistribution at 4 and $24 \mathrm{~h}$ after injection into a MI mouse model.

Results: MSC-derived exosomes were successfully labeled by glucose modified gold nanoparticles and CT imaging of these labeled exosomes indicated that MSC-Exo remained in the MI area for up to $24 \mathrm{~h}$ after intramyocardial injection. Additionally, few MSC-Exo were observed in some other organs, particularly the liver, spleen, and kidney.

Conclusions: A gentle method was used for loading GNPs into exosomes, and their successful labeling without causing aggregation was verified. In vivo CT imaging revealed the retention of MSC-Exo in the MI area, indicating their usefulness for improving heart function after infarction.

Keywords: Myocardial infarction (MI); exosomes; mesenchymal stem cells (MSCs); in vivo imaging

Submitted Feb 03, 2021. Accepted for publication Mar 24, 2021.

doi: $10.21037 /$ atm-21-981

View this article at: http://dx.doi.org/10.21037/atm-21-981

\section{Introduction}

In developed countries, cardiovascular disease is the leading cause of morbidity and mortality, resulting in an extremely high economic burden (1). For example, the costs for cardiovascular disease-related care were
$\$ 315.4$ billion in 2010 , and this number is projected to be $\$ 918$ billion by 2030 , as $43.9 \%$ of the population is predicted to suffer cardiovascular disease $(1,2)$. Among cardiovascular diseases, acute myocardial infarction (MI), which is defined pathologically as myocardial cell death due 
to prolonged ischemia, is the main contributor. Typically, after MI, the damaged and relatively non-regenerative myocardium undergoes a degeneration and reconstruction process, causing heart failure and ultimately death.

Mesenchymal stem cells (MSCs) have been shown to repair infarcted myocardium by activating endogenous tissue repair through paracrine signaling as well as exhibiting immunomodulatory properties, reducing immune-mediated damage after MI $(3,4)$. However, their safety and effectiveness have been widely concerning in clinical practice, with contamination related to cell transplantation, cell death, and immune rejection being the main problems (5-7). Therefore, alternative treatment strategies must be developed $(8,9)$. Numerous studies have shown that the therapeutic effect of MSCs is due to their paracrine effects, and MSC-derived exosomes (MSCExo) can also improve heart function after infarction while avoiding the problems caused by MSCs (10-13).

The term exosome [which differs from the exosome complex involved in RNA degradation (14)] was initially used to describe vesicles secreted by various cell types that showed 5'nucleotidase activity (15). Subsequently, the term exosome was adopted for small $(30-100 \mathrm{~nm})$ vesicles of endosomal origin that are released during reticulocyte differentiation (16). Exosomes are actually intraluminal vesicles formed by the inward budding of the endosomal membrane during the maturation of multivesicular endosomes (MVE), which are intermediates within the endosomal system, and are secreted after the fusion of MVE with the cell surface $(17,18)$. Exosomes were first found to be vehicles for disposing of unwanted transferrin, and received little attention for many years (19). In the mid-1990s, it was reported that exosomes were secreted by B lymphocytes and dendritic cells with potential functions in connection with immune regulation, and were considered to be carriers of anti-tumor immune responses $(20,21)$. At present, exosome secretion has been observed in most mammalian cells, and the significance of exosome vesicles lies in their capacity to transfer information to other cells to modify the recipient cell's function (22). Proteins, genetic material (including, mRNAs, miRNAs, lncRNAs), metabolites, and lipids are selectively recruited and loaded into exosomes, which deliver these cargos to recipient cells and thus act as vehicles of intercellular communication under both physiological and pathological conditions (23-26). Furthermore, exosomes possess an intrinsic ability to cross biological barriers. Based on this, researchers pay considerable attention to developing exosome-based therapeutic strategies for constructing natural drug delivery vehicles loaded with a desired therapeutic molecule (27).

Exosomes can deliver miRNA that might be useful to limit tissue injury, improve neovascularization, and prevent subsequent negative cardiac remodeling that can lead to heart failure. However, few studies have directly compared the therapeutic effects of MSCs and MSC-Exo so far. Therefore, it is uncertain whether MSCs can be replaced by MSC-Exo for cardiac repair. Additionally, the molecular mechanisms behind MSC- and MSC- Exo-mediated cardiac repair are unknown. Moreover, the trafficking mechanisms, in vivo biodistribution, and pharmacokinetics of exogenously administered MSC-Exo must be revealed before it can be used as a therapeutic agent (28). In vivo imaging of the exosomes can provide important information for explaining these issues (29-31). Among the imaging modalities, optical imaging has been widely used for tracking and analyzing a series of biomolecules; however, the limited ability to visualize within deep sites confines its application in living animals $(32,33)$. CT is a non-invasive imaging method that can take images of deep structures in the body and maybe useful for exosome tracking in vivo. However, exosomes cannot be detected by CT directly, and CT contrast agents must be added to label exosomes for visualization by CT. Advances in nanotechnological techniques have enabled the use of ultra-small gold nanoparticles (GNPs) of several nanometers in size for labeling. And for their strong X-ray attenuation, a high degree of flexibility in terms of functional groups for coating and targeting, and having been proved to be nontoxic and biocompatible in vivo, GNPs are ideal contrast agents for in vivo CT imaging and tracking of exosomes $(34,35)$. Owing to their minute size, these nanoparticles can be easily applied to label nanovesicles such as exosomes (36).

For exosome labeling, an indirect strategy is typically employed, wherein GNPs are added into the parent cell culture medium and are taken up by cells through endocytic pathways. Some of the contrast agents may load into the exosomes during their biogenesis, and the secreted exosomes are then isolated from the cell culture supernatant $(37,38)$. However, this indirect strategy results in only a small fraction of GNPs loading into the exosomes $(39,40)$. Some direct strategies are used to introduce GNPs, generally by instantaneous destruction of the exosomal membrane, however this may damage the integrity and functionality of the exosomes.

In the present study, we developed a gentle direct method for loading GNPs into isolated MSC-Exo without 
damaging the exosomal membrane. A MI mouse model was established by ischemia surgery, and GNP-labeled exosomes were injected for in vivo CT imaging of trafficking and in vivo distribution analyses in living mice.

We present the following article in accordance with the ARRIVE reporting checklist (available at http://dx.doi. org/10.21037/atm-21-981).

\section{Methods}

\section{Cell culture}

Mouse MSCs were purchased from Cyagen Biosciences (Guangzhou, China). The cells were maintained in a culture medium in a humidified incubator with $5 \%$ carbon dioxide $\left(\mathrm{CO}_{2}\right)$ at $37^{\circ} \mathrm{C}$, and routinely detected and verified to be free of mycoplasma contamination. Before collecting the cell culture supernatant for exosome isolation, fetal bovine serum (FBS, Biological Industries, Beit-Haemek, Israel) was first depleted of exosomes. The exo-depleted FBS was prepared by ultracentrifugation at $120,000 \times \mathrm{g}$ for $18 \mathrm{~h}$ at $4{ }^{\circ} \mathrm{C}$, and then, the supernatant was filtered through a $0.22-\mu \mathrm{m}$ filter unit. Complete culture medium was prepared by supplementing $500 \mathrm{~mL}$ of Dulbecco's modified eagle medium (DMEM) (Gibco, Grand Island, NY, USA) with $50 \mathrm{~mL}$ of exo-depleted FBS, $5 \mathrm{~mL}$ of $100 \times$ L-glutamine (Amresco, Dallas, TX, USA), and $2.5 \mathrm{~mL}$ of 100x Penicillin-Streptomycin Solution (Solarbio, Beijing, China). Approximately $3 \times 10^{6} \mathrm{C} 57 \mathrm{BL} / 6 \mathrm{MSCs}$ in $30 \mathrm{~mL}$ of complete medium were seeded into each $\mathrm{P} 150$ tissue culture plate. The cells were cultured for $72 \mathrm{~h}$ before supernatant collection.

\section{Exosome isolation}

Exosomes were isolated by collecting the cell culture supernatant into $50 \mathrm{~mL}$ Falcon tubes and centrifugation with $1,000 \times g$ at $10^{\circ} \mathrm{C}$ for 5 min using a table-top centrifuge (TDZ4-WS/TDZ4WS, Cence, Istanbul, Turkey). The supernatant was pipetted into ultracentrifuge tubes $(65 \mathrm{~mL} /$ tube) and centrifuged at $15,000 \times \mathrm{g}$ for $45 \mathrm{~min}$ at $4{ }^{\circ} \mathrm{C}$ to deplete apoptotic bodies, cell debris, and large microvesicles. A Type 45-Ti ultracentrifuge rotor (prechilled at $4{ }^{\circ} \mathrm{C}$; Beckman Coulter, Brea, CA, USA) was used for this and the following process. The supernatant was subsequently filtered through a $0.22-\mu \mathrm{m}$ filter and centrifuged at $120,000 \times \mathrm{g}$ for $90 \mathrm{~min}$ at $4^{\circ} \mathrm{C}$ to isolate the exosomes. After the supernatant was carefully discarded, the pellets were resuspended in several milliliters of ice-cold phosphate-buffered saline (PBS) in each tube. As for the exosome washing process, all of the samples were combined into one tube and the final volume was added to $65 \mathrm{~mL}$ with ice-cold PBS, and then centrifuged at 120,000 $\times \mathrm{g}$ for another $90 \mathrm{~min}$ at $4{ }^{\circ} \mathrm{C}$. The purified exosomes collected into the pellet were resuspended in $200 \mu \mathrm{L}$ of sterilized PBS.

\section{Exosome characterization}

The size and concentration of exosomes were analyzed by nanoparticle tracking analysis (NTA, Zetaview, Particle Matrix). For NTA measurement, $5 \mu \mathrm{L}$ samples were diluted $(1,000 \times)$ to a volume of $5 \mathrm{~mL}$ in PBS. Next, the diluted sample was injected into the sample chamber using a disposable sterile syringe. Transmission electron microscopy (TEM) was used to observe the morphology and size of the exosomes. A $5 \mu \mathrm{L}$ exosome suspension was dropped on a 400-mesh copper grid and incubated for $20 \mathrm{~min}$. After washing with PBS and ultrapure water several times, the sample was subjected to negative staining by adding $20 \mu \mathrm{L}$ of $2 \%$ uranyl acetate, and incubated for $10 \mathrm{~min}$. The exosome samples were observed by TEM using a JEM 2100F (JEOL, Tokyo, Japan).

\section{Surface modification of GNPS}

GNPs were purchased from BBI Solutions (Crumlin, UK). The glucose-modified GNPs were prepared as previously described with some modifications (41-43). Briefly, $4 \mathrm{~mL}$ GNP solution was filtered through Amicon Ultra-15 $100 \mathrm{kDa}$ centrifugal filter devices (Millipore, Billerica, MA, USA), and ultrafiltration was carried out at 5,000 $\times \mathrm{g}$ for $10 \mathrm{~min}$. The GNPs were then resuspended in $4 \mathrm{~mL}$ deionized water. This process was repeated twice to remove the excess coating of polymers in the GNP solution. Next, $200 \mu \mathrm{L} 1 \mathrm{mM}$ glucose-monofunctional polyethylene glycolthiol (glucose-mPEG-SH) was added to $1 \mathrm{~mL} 0.3 \mathrm{mM}$ GNP solution. The mixture was stirred for $24 \mathrm{~h}$ at room temperature $\left(25^{\circ} \mathrm{C}\right)$ and ultrafiltration was conducted as described above to remove excess glucose-mPEG-SH.

\section{GNP characterization}

TEM was used to evaluate the shape and size of the GNPs, and these results were further verified by dynamic light scattering. The average particle size and zeta potential of 
the prepared GNPs were analyzed with a Zetasizernano ZS90 (Malvern, Ltd., Malvern, UK). The size and zeta potential were measured at room temperature, and the refractive index and viscosity of water were used to calculate the results.

\section{Exosomes labeled with glucose-coated GNPs}

Since the exosomal membrane was similar to the parent cell membrane in terms of topology and structural features, it was possible to label exosomes directly rather than by employing indirect strategies through the parent cell takenup process (44). $100 \mu \mathrm{L}$ exosomes suspended in PBS were mixed and then incubated with $100 \mu \mathrm{L} 0.3 \mathrm{mM}$ glucosecoated GNPs for 3 or $10 \mathrm{~h}$ at $37^{\circ} \mathrm{C}$. The separation of exosomes from unlabeled GNPs was achieved by density gradient ultracentrifugation.

\section{Density gradient ultracentrifugation}

Density gradient ultracentrifugation was conducted to purify the exosomes and remove excess GNPs that failed to enter the exosomes. The density of exosomes was $1.15-1.19 \mathrm{~g} / \mathrm{mL}$, and the density of GNPs was estimated to be $2.4 \mathrm{~g} / \mathrm{mL}$. Three sucrose solutions $(10 \mathrm{~mL}$ each), with concentrations of $25 \%, 45 \%$, and $60 \%$ were prepared at densities of $1.1036,1.2025$, and 1.2865 , respectively. Ultraclean centrifuge tubes with a volume of $4 \mathrm{~mL}$ were used. First, $1 \mathrm{~mL} 25 \%$ sucrose solution was injected into the tube, and then $1 \mathrm{~mL} \mathrm{45 \%} \mathrm{sucrose} \mathrm{solution} \mathrm{was} \mathrm{injected} \mathrm{into}$ the bottom layer under the initial $25 \%$ solution with a long syringe needle. Subsequently, the $60 \%$ sucrose solution was similarly injected into the bottom layer under the $45 \%$ solution. The samples were then placed on top of the $25 \%$ solution. Ultracentrifugation was conducted at 150,000 $\times \mathrm{g}$ for $4 \mathrm{~h}$ at $4{ }^{\circ} \mathrm{C}$ in an SW60 ultracentrifuge rotor (pre-chilled at $4{ }^{\circ} \mathrm{C}$; Beckman Coulter).

\section{Ischemia surgical and exosome administration}

C57 BL/6 male mice (8 weeks, 25-30 g) were conditioned in a $12: 12 \mathrm{~h}$ light/dark cycle with free access to fodder and water, under a fixed temperature $\left(23^{\circ} \mathrm{C}\right)$ and humidity $(50 \%)$. The mouse model of MI was established as previously described $(45,46)$. Eight-week-old male mice were anesthetized via intraperitoneal injection using mixture of ketamine and pentobarbital sodium. The mice were then weighed and the weight was recorded, and their chest walls were shaved prior to the surgery. The mice were placed in the supine position, and tracheal intubation was performed under a direct laryngoscopy. A small animal respirator was used to assist the breathing of the mice during the surgery. Correct intubation was confirmed by observing the expansion and contraction of the chest during assisted breathing. The ischemia surgical operation was carried out by ligating the left coronary artery using a $10-\mathrm{cm} 7-0$ silk suture through a $15-\mathrm{mm}$ opening at the fourth intercostal space. A plain knot was tied and the mice were left in situ for $10 \mathrm{~min}$. Accomplishment of ischemia was verified by the appearance of discoloration of the heart surface. For intramyocardial injection, $20 \mu \mathrm{L}$ of labeled exosomes with a total number of approximately $2.8 \times 10^{9}$ were administered slowly at three different sites around the discolored area. The chest cavity was carefully closed in layers, and the mice were gradually weaned from the respirator. The endotracheal tube was removed once spontaneous respiration resumed, and the mice were then recovered under a heating lamp. The mice were maintained in a supervised setting until completely conscious, and were then provided with free access to standard chow and water after returning to their cages.

Experiments were performed under a project license (No. SYXK(HU)2018-0029) granted by Laboratory Animal Welfare Ethics Committee of Shanghai University of Medical and Health, in compliance with Chinese national or institutional guidelines for the care and use of animals.

\section{CT imaging}

In vivo CT imaging of the whole body was performed using a micro-CT scanner (PINGSENG, NEMO ${ }^{\circledR}$ Micro CT, KunShan, China) with a nominal resolution of $7.5 \mu \mathrm{m}$, $1 \mathrm{~mm}$ aluminum filter, and a tube voltage of $70 \mathrm{kV}$. The mice were scanned at $4 \mathrm{~h}$ and $24 \mathrm{~h}$ after injection of the exosomes. Reconstruction was conducted with an ordered subset expectation maximization iteration algorithm accelerated by graphics processing unit (GPU). Ring artifact reduction and scatter correction were applied.

\section{Statistical analysis}

Data are presented as mean \pm SEM. All statistical analyses were performed with Statistical Product and Service Solutions (SPSS) software (version 17.0, SPSS, USA), and $\mathrm{P}<0.05$ was considered statistically significant. 

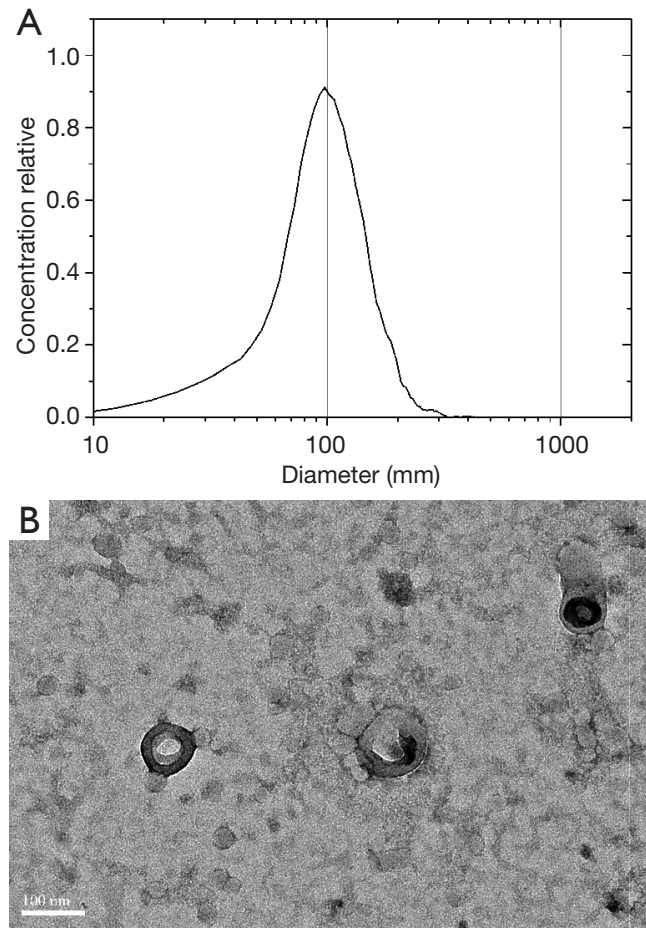

Figure 1 The characterization results of isolated exosomes. The NTA result of the exosomes (A). And the TEM image with a scale bar of $100 \mathrm{~nm}$ of the exosomes (B). NTA, nanoparticle tracking analysis; TEM, transmission electron microscopy.

\section{Results}

\section{Characterization of exosomes}

The cell culture supernatant $(180 \mathrm{~mL})$ was collected and subjected to ultracentrifugation to isolate the exosomes. Following this process, some sediment was visible by direct observation. The sample size was measured to be $97.7 \mathrm{~nm}$ (Figure $1 A$ ) by NTA, which was also confirmed by TEM analysis (Figure 1B). The sample concentration was approximately $2.3 \times 10^{11}$ particles $/ \mathrm{mL}$.

\section{Surface modification of GNPs}

Glucose-modified GNPs were prepared by mixing glucosemPEG-SH with GNPs. After reacting with the thiol group of glucose-mPEG-SH on the surface of GNPs, the original coating polymers were washed off by ultrafiltration. TEM revealed the same gold core size for both the original and glucose-modified GNPs, while the distance among the GNPs increased after surface modification (Figure $2 A, B$ ), indicating that the glucose-mPEG-SH had a longer chain
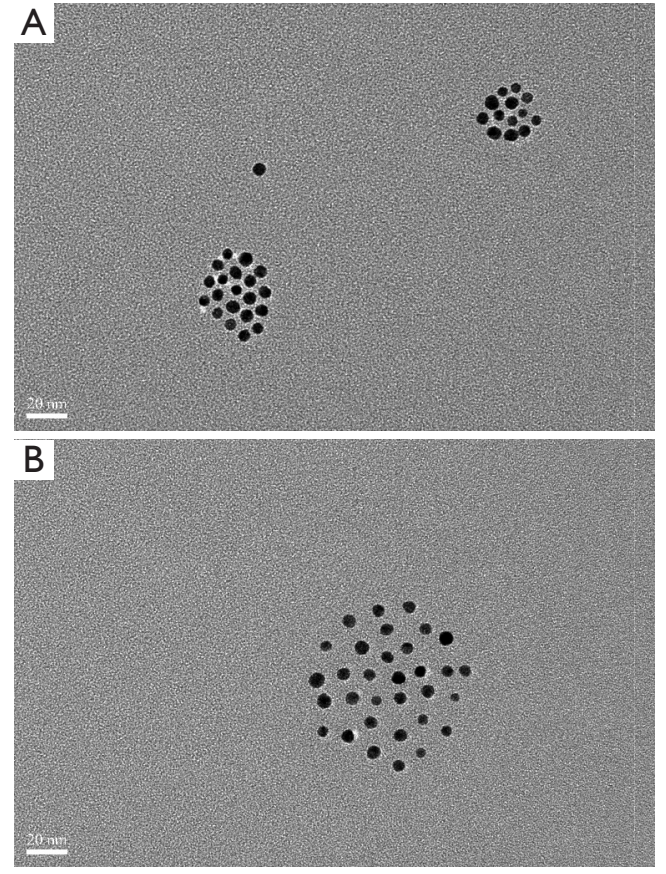

Figure 2 TEM images of the GNPs. TEM image of the original GNPs (A). And TEM image of the glucose-modified GNPs (B). TEM, transmission electron microscopy; GNPs, gold nanoparticles.

Table 1 Average size and zeta potential of original and glucose-modified GNPs

\begin{tabular}{lcc}
\hline & Size $(\mathrm{nm})$ & Zeta potential \\
\hline Original GNPs & $7.5 \pm 0.2$ & $-23.4 \pm 2$ \\
Glucose-modified GNPs & $32.5 \pm 1$ & $-6.2 \pm 0.4$ \\
\hline
\end{tabular}

GNPs, gold nanoparticles.

than the original coating polymers. In addition, TEM indicated that glucose-modified GNPs were prepared without forming aggregates. The sizes and zeta-potentials of the resulting GNPs were measured (Table 1). The average size of the original GNPs was $\sim 7.5 \mathrm{~nm}$, and increased to $\sim 32.5 \mathrm{~nm}$ after surface modification, which concurred with the TEM results. The zeta potential results indicated that glucose-modified GNPs had a more neutral surface.

\section{GNPs labeling of exosomes}

Exosomes were incubated with glucose-coated GNPs for 3 or $10 \mathrm{~h}$ at $37^{\circ} \mathrm{C}$ to load GNPs into exosomes. The particle ratio of GNPs and exosomes was approximately 


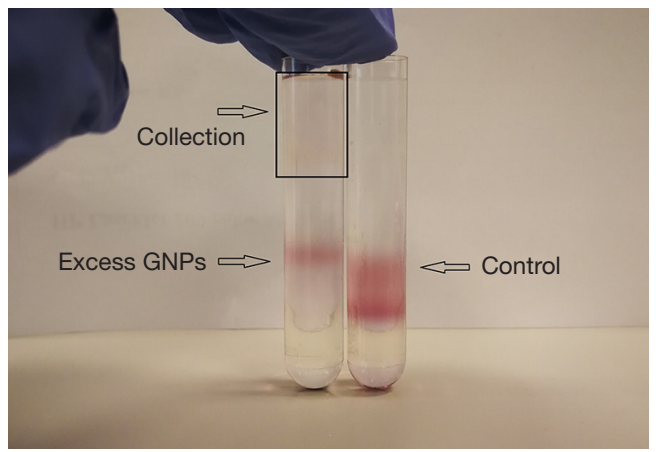

Figure 3 Exosome samples (left) and GNPs (right) after density gradient ultracentrifugation. GNPs, gold nanoparticles.

200:1 during the incubation process, and GNPs that failed to enter the exosomes were isolated by density gradient ultracentrifugation. A sample of GNPs alone was used as a control during density gradient ultracentrifugation. Excess GNPs in the exosome sample were detected at the same level as the control in the gradient, as shown in Figure 3. GNPs were not detected at the bottom of the tube, possibly because the centrifugation time was too short.

After loading the GNPs into the exosomes, NTA was conducted for size measurement and aggregate detection. The results showed that the curve shapes were very similar to each other, exhibiting only a slight shift, which indicated an increase in size (Figure 4A,B,C). This size increase may have been caused by internalization of the GNPs. No obvious peaks indicating large-size particles were observed, indicating that aggregation of the exosomes was limited. The size and shape of the curves was consistent with those reported previously (44).

TEM imaging depicted an obvious dark spot, which was confirmed as GNPs, in the same location as that of the exosomes (Figure 5). Thus, the GNPs appeared to have entered the exosomes.

\section{In vivo CT imaging}

GNP-labeled exosomes were injected at three different points around the discoloration area after ischemia surgery. In vivo CT imaging was conducted at $4 \mathrm{~h}$ (Figure $6 \mathrm{~A}$ ) and $24 \mathrm{~h}$ (Figure $6 B)$ after injection of the exosomes. GNPs were obvious in the CT images (red area) both at 4 and $24 \mathrm{~h}$, and the red area was found to have expanded after $24 \mathrm{~h}$ compared to at $4 \mathrm{~h}$, indicating that MSC-Exo remained in the myocardium for up to $24 \mathrm{~h}$. Additionally, some other organs, particularly the liver, spleen, and kidney, were monitored to detect
Gong et al. Visualizing gold nanoparticle labeled exosomes in vivo
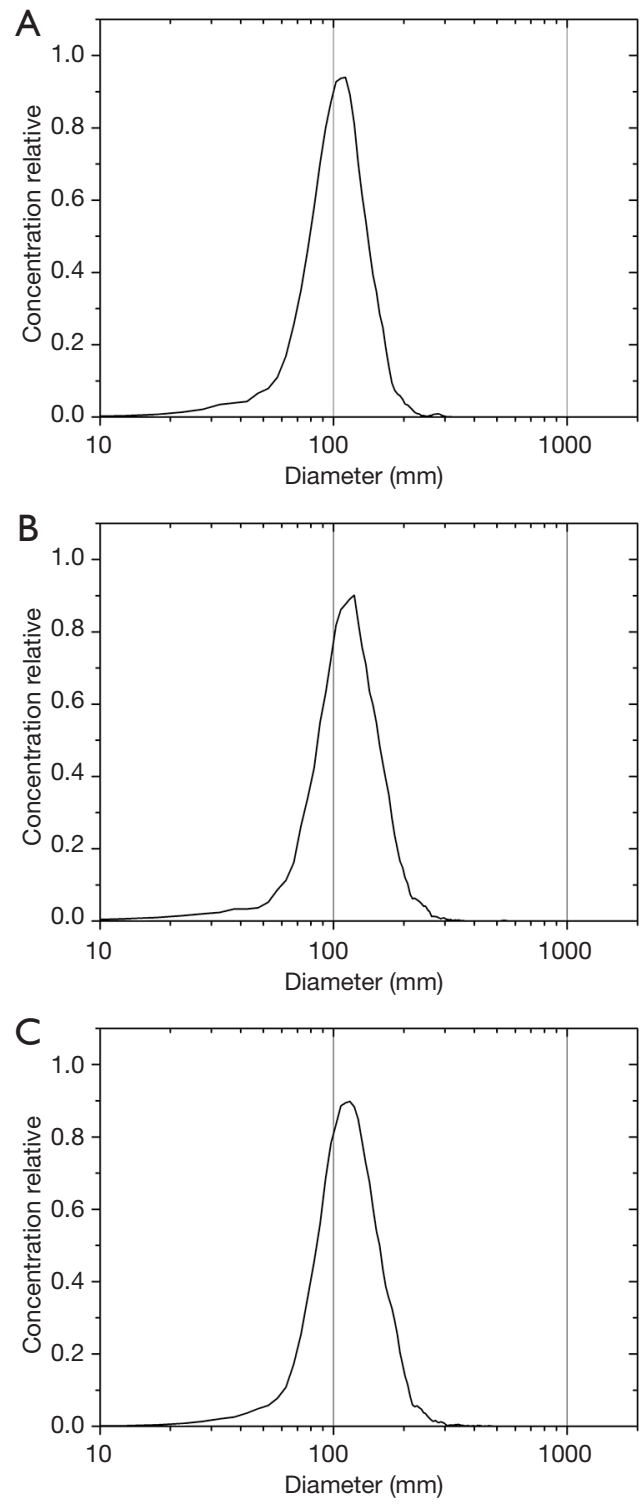

Figure 4 NTA results of exosomes with different incubation conditions. NTA results of exosomes incubated without GNPs for $3 \mathrm{~h}$ at $37{ }^{\circ} \mathrm{C}$ (A). NTA results of exosomes incubated with GNPs for $3 \mathrm{~h}$ at $37{ }^{\circ} \mathrm{C}(\mathrm{B})$. And NTA results of exosomes incubated with GNPs for $10 \mathrm{~h}$ at $37^{\circ} \mathrm{C}(\mathrm{C})$. NTA, nanoparticle tracking analysis; GNPs, gold nanoparticles.

GNPs. However, few GNPs were observed in these organs, demonstrating that most GNPs remained in the MI area.

\section{Discussion}

Due to the low efficiency of the indirect approach for loading 


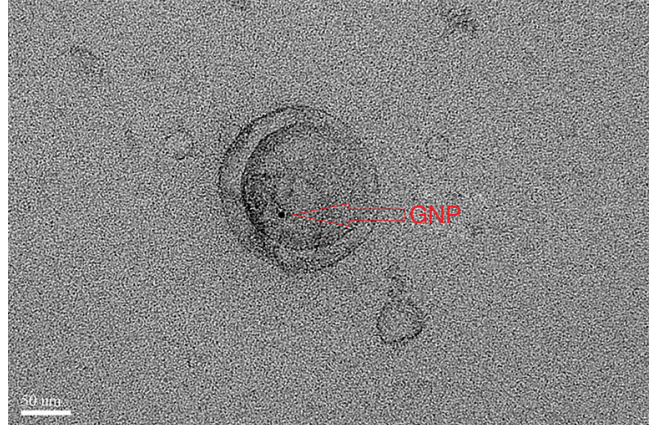

Figure 5 TEM image of exosomes incubated with GNPs for $10 \mathrm{~h}$ at $37^{\circ} \mathrm{C}$. GNPs, gold nanoparticles.

the GNPs, several direct labeling methods have been reported for loading contrast agents into purified exosomes directly. For example, electroporation is an efficient method of loading exosomes with drugs (47), RNA cargo (48-50), or $5 \mathrm{~nm}$ superparamagnetic iron oxide nanoparticles (51). A long electric pulse is used in electroporation to temporarily disturb the phospholipid bilayer, and gives rise to temporary pores in the membranes that permit molecules to pass into the exosomes (31). However, electroporation may increase exosome aggregation and damage the integrity of the exosomes. A gentle method was used in this study that maintains the dispersant ability and integrity of exosomes during the labeling process.

Exosomes were incubated with glucose-coated GNPs for 3 or $10 \mathrm{~h}$ at $37^{\circ} \mathrm{C}$, as GNP internalization is known to be energy dependent and $37^{\circ} \mathrm{C}$ is more suitable than a low temperature (44). The internalization process is also associated with the glucose transporter (GLUT-1), and thus glucose modification of GNPs is necessary. Excess free glucose should be washed off to prevent occupation and saturation of GLUT-1 at the exosome surface, which would prevent internalization (44). Ultrafiltration was conducted to remove free glucose. Similarly, excess GNPs were removed by density gradient centrifugation after coincubating of GNPs and exosomes, avoiding the effect of unlabeled gold nanoparticles on CT imaging results.

The NTA results of the exosomes before and after the GNPs labeling changed little, and no large particles appeared after labeling, indicating that the labeling process did not cause the agglomeration of exosomes. The TEM images confirmed this result, meanwhile, the colocalization of GNPs and exosomes was also observed, indicating the successful labeling of GNPs.

The retention of exosomes in the myocardium was

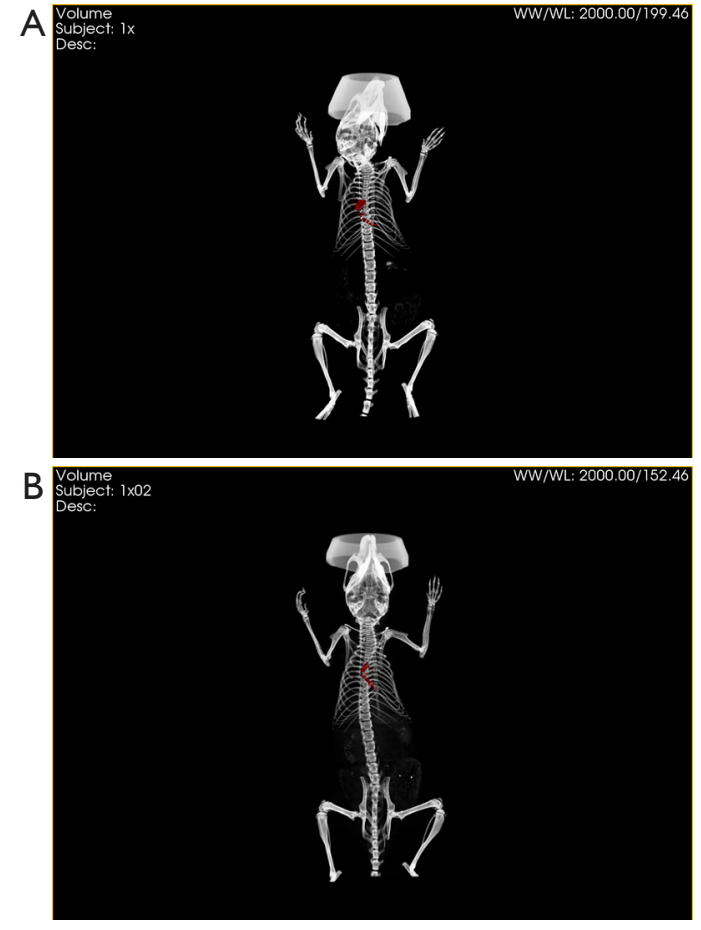

Figure 6 In vivo CT imaging at different time after injection. In vivo CT imaging at $4 \mathrm{~h}$ after injection (A). And In vivo CT imaging at $24 \mathrm{~h}$ after injection (B).

observed at 4 and $24 \mathrm{~h}$ after the injection, indicating that the exosomes injected into the myocardium can stay for enough time and may help repair the damaged myocardium. Nonetheless, additional studies are necessary to determine the exact mechanisms by which MSC-derived exosomes improve heart function. And the results also show that the GNPs labeling can be used for studying the in vivo biodistribution and pharmacokinetics of exogenously administered exosomes.

\section{Conclusions}

In this study, a gentle method was used for loading GNPs into exosomes, and their successful labeling without causing aggregation was verified. In vivo CT imaging revealed the retention of MSC-Exo in the MI area, indicating their usefulness for improving heart function after infarction. The ability to track exosomes in the hearts of living bodies using conventional CT approaches significantly advances translational exosome science. Thus, it may be possible to conduct high-resolution, sensitive, and non-invasive tracking of exosomal nanomedicines for $\mathrm{MI}$ applications. 


\section{Acknowledgments}

Funding: The work was supported by the Shanghai Municipal Education Commission-Plateau Disciplinary Program for Medical Technology of SUMHS, 2018-2020 (China), the National Natural Science Foundation of China (No. 81660284 and 81860316), and the Key R \& D projects of Jiangxi Province (No. 20192BBGL70035).

\section{Footnote}

Reporting Checklist: The authors have completed the ARRIVE reporting checklist. Available at http://dx.doi. org/10.21037/atm-21-981

Data Sharing Statement: Available at http://dx.doi. org/10.21037/atm-21-981

Conflicts of Interest: All authors have completed the ICMJE uniform disclosure form (available at http://dx.doi. org/10.21037/atm-21-981). All authors report funding from Shanghai Municipal Education CommissionPlateau Disciplinary Program for Medical Technology of SUMHS, 2018-2020 (China), the National Natural Science Foundation of China (No. 81660284 and 81860316), and the Key R \& D projects of Jiangxi Province (No. 20192BBGL70035). The authors have no other conflicts of interest to declare.

Ethical Statement: The authors are accountable for all aspects of the work in ensuring that questions related to the accuracy or integrity of any part of the work are appropriately investigated and resolved. Experiments were performed under a project license (No.: SYXK(HU)2018-0029) granted by Laboratory Animal Welfare Ethics Committee of Shanghai University of Medical and Health, in compliance with Chinese national or institutional guidelines for the care and use of animals.

Open Access Statement: This is an Open Access article distributed in accordance with the Creative Commons Attribution-NonCommercial-NoDerivs 4.0 International License (CC BY-NC-ND 4.0), which permits the noncommercial replication and distribution of the article with the strict proviso that no changes or edits are made and the original work is properly cited (including links to both the formal publication through the relevant DOI and the license). See: https://creativecommons.org/licenses/by-nc-nd/4.0/.

\section{References}

1. Go AS, Mozaffarian D, Roger VL, et al. Executive summary: heart disease and stroke statistics--2014 update: a report from the American Heart Association. Circulation 2014;129:399-410.

2. Gray WD, French KM, Ghosh-Choudhary S, et al. Identification of therapeutic covariant microRNA clusters in hypoxia-treated cardiac progenitor cell exosomes using systems biology. Circ Res 2015;116:255-63.

3. Uemura R, Xu M, Ahmad N, et al. Bonemarrow stem cells prevent left ventricular remodeling of ischemic heart through paracrine signaling. Circ Res 2006;98:1414-21.

4. Rosenberg M, Lutz M, Kuhl C, et al. Coculture with hematopoietic stem cells protects cardiomyocytes against apoptosis via paracrine activation of AKT. J Transl Med 2012;10:115.

5. Hare JM, Traverse JH, Henry TD, et al. Dose-Escalation Study of Intravenous Adult Human Mesenchymal Stem Cells (Prochymal) After Acute Myocardial Infarction. J Am Coll Cardiol 2009;54:2277-86.

6. Sanganalmath SK, Bolli R. Cell therapy for heart failure: a comprehensive overview ofexperimental and clinical studies, current challenges, and future directions. Circ Res 2013;113:810-34.

7. Golpanian S, DiFede DL, Pujoletal MV. Rationale and design of the allogeneiC human mesenchymal stem cells (hMSC) in patients with aging fRAilTy via intravenoUS delivery (CRATUS) study: a phase I/II, randomized, blinded and placebo controlled trial to evaluate the Safety and potential efficacy of allogeneic human mesenchymal stem cell infusion in patients with aging frailty. Oncotarget 2016;7:11899-912.

8. Boon RA, Dimmeler S. MicroRNAs in myocardial infarction. Nat Rev Cardiol 2015;12:135-42.

9. Li Y, Shen Z, Yu XY. Transport of microRNAs via exosomes. Nat Rev Cardiol 2015;12:198.

10. Teng X, Chen L, Chen W, et al. Mesenchymal stem cell-derived exosomes improve the microenvironment ofinfarctedmyocardium contributing to angiogenesis and anti-inflammation. Cell. Physiol. Biochem 2015;37:2415-24.

11. Lai RC, Arslan F, Lee MM, et al. Exosome secreted by MSC reduces myocardial ischemia/reperfusion injury. Stem Cell Res 2010;4:214-22.

12. Bian S, Zhang L, Duan L, et al. Extracellular vesicles derived from human bone marrow mesenchymal stem cells promote angiogenesis in a rat myocardial infarction model. 
J Mol Med 2014;92:387-97.

13. Shao L, Zhang Y, Lan B, et al. MiRNA-Sequence Indicates That Mesenchymal Stem Cells and Exosomes Have Similar Mechanism to Enhance Cardiac Repair. BioMed Res Int 2017;2017:4150705.

14. Wasmuth EV, Januszyk K, Lima CD. Structure of an Rrp6-RNA exosome complex bound to poly(A) RNA. Nature 2014;511:435-9.

15. Trams EG, Lauter CJ, Salem NJ, et al. Exfoliation of membrane ecto-enzymes in the form of micro-vesicles. Biochim Biophys Acta 1981;645:63-70.

16. Johnstone RM, Adam M, Hammond JR, et al. Vesicle formation during reticulocyte maturation. Association of plasma membrane activities with released vesicles (exosomes). J Biol Chem 1987;262:9412-20.

17. Harding C, Heuser J, and Stahl P. Endocytosis and intracellular processing of transferrin and colloidalgold transferrin in rat reticulocytes: demonstration of a pathway for receptor shedding. Eur J Cell Biol 1984;35:256-63.

18. Pan BT, Teng K, Wu C, et al. Johnstone, Electron microscopic evidence for externalization of the transferrin receptor in vesicular form in sheep reticulocytes. J Cell Biol 1985;101:942-8

19. Pan BT, Johnstone RM. Fate of the transferrin receptor during maturation of sheep reticulocytes in vitro: selective externalization of the receptor. Cell 1983;33:967-78.

20. Raposo G, Nijman HW, Stoorvogel W, et al. B lymphocytes secrete antigen-presenting vesicles. J Exp Med 1996;183:1161-72.

21. Zitvogel L, Regnault A, Lozier A, et al. Eradication of established murine tumors using a novel cell-free vaccine: dendritic cell-derived exosomes. Nat Med 1998;4:594-600.

22. Yáñez-Mó M, Siljander PR, Andreu Z, et al. Biological properties of extracellular vesicles and their physiological functions. J. Extracell. Vesicles 2015;4:27066.

23. Balaj L, Lessard R, Dai L, et al. Tumour microvesicles contain retrotransposon elements and amplified oncogene sequences. Nat Commun 2011;2:180.

24. Choi DS, Kim DK, Kim YK, et al. Proteomics, transcriptomics and lipidomics of exosomes and ectosomes. Proteomics 2013;13:1554-71.

25. Thakur BK, Zhang H, Becker A, et al. Double-stranded DNA in exosomes: a novel biomarker in cancer detection. Cell Res 2014;24:766-9.

26. Tetta C, Ghigo E, Silengo L, et al. Extracellular vesicles as an emerging mechanism of cell-to-cell communication. Endocrine 2013;44:11-9.

27. Zhang H, Freitas D, Kim HS, et al. Identification of distinct nanoparticles and subsets of extracellular vesicles by asymmetric flow field-flow fractionation. Nat Cell Biol 2018;20:332-43.

28. Takahashi $Y$, Nishikawa M, Shinotsuka H, et al. Visualization and in vivo tracking of the exosomes of murine melanoma B16-BL6 cells in mice after intravenous injection. J Biotechnol 2013;165:77-84.

29. Johnsen KB, Gudbergsson JM, Skov MN, et al. A Comprehensive Overview of Exosomes as Drug Delivery Vehicles - Endogenous Nanocarriers for Targeted Cancer Therapy. Biochim Biophys Acta 2014;1846:75-87.

30. Lai RC, Yeo RWY, Tan KH, et al. Exosomes for Drug Delivery - A Novel Application for the Mesenchymal Stem Cell. Biotechnol Adv 2013;31:543-51.

31. Busato A, Bonafede R, Bontempi P, et al. Magnetic resonance imaging of ultrasmall superparamagnetic iron oxide-labeled exosomes from stem cells: a new method to obtain labeled exosomes. Int J Nanomedicine 2016;11:2481-90.

32. Yang T, Martin P, Fogarty B, et al. Exosome Delivered Anticancer Drugs across the Blood-Brain Barrier for Brain Cancer Therapy in Danio Rerio. Pharm. Res 2015;32:2003-14.

33. Pasternak O, Kubicki M, Shenton ME. In Vivo Imaging of Neuroinflammation in Schizophrenia. Schizophr Res 2016;173:200-12.

34. Arvizo R. Gold Nanoparticles: Opportunities and Challenges in Nanomedicine. Expert Opin Drug Deliv 2010;7:753-63.

35. Reuveni T, Motiei M, Romman Z, et al. Targeted Gold Nanoparticles Enable Molecular CT Imaging of Cancer: An in Vivo Study. Int J Nanomedicine 2011;6:2859-64.

36. Busato A, Bonafede R, Bontempi P, et al. Labeling and Magnetic Resonance Imaging of Exosomes Isolated from Adipose Stem Cells. Curr Protoc Cell Biol 2017;75:3.44.1-15.

37. Sun D, Zhuang X, Xiang X, et al. A Novel Nanoparticle Drug Delivery System: The Anti-Inflammatory Activity of Curcumin Is Enhanced When Encapsulated in Exosomes. Mol Ther 2010;18:1606-14.

38. Haney MJ, Klyachko NL, Zhao Y, et al. Exosomes as Drug Delivery Vehicles for Parkinson's Disease Therapy. J Control Release 2015;207:18-30.

39. Alhasan AH, Patel PC, Choi CHJ, et al. Exosome Encased Spherical Nucleic Acid Gold Nanoparticle Conjugates as Potent microRNA Regulation Agents. Small 2014;10:186-92.

40. Roma-Rodrigues C, Pereira F, Alves de Matos AP, et al. 


\section{Page 10 of 10}

Smuggling Gold Nanoparticles across Cell Types - a New Role for Exosomes in Gene Silencing. Nanomedicine 2017;13:1389-98.

41. Niidome T, Yamagata M, Okamoto Y, et al. PEGmodified gold nanorods with a stealth character for in vivo applications. J Control Release 2006;114:343-7.

42. Eghtedari M, Liopo AV, Copland JA, et al. Engineering of hetero-functional gold nanorods for the in vivo molecular targeting of breast cancer cells. Nano Lett 2009;9:287-91.

43. Jansen ED, Liopo A, Thomas RJ, et al. Photothermal therapy of acute leukemia cells in the near-infrared region using gold nanorods CD-33 conjugates. Proc SPIE - The International Society for Optical Engineering 2011;7897:789710.

44. Betzer O, Perets N, Angel A, et al. In Vivo Neuroimaging of Exosomes Using Gold Nanoparticles. ACS Nano 2017;11:10883-93.

45. Patten RD, Aronovitz MJ, Deras-Mejia L, et al. Ventricular remodeling in a mouse model of myocardial infarction. Am J Physiol 1998;274:H1812-20.

46. Vandergriff A, Huang K, Shen D, et al. Targeting

Cite this article as: Gong L, Weng Y, Zhou W, Zhang K, Li W, Jiang J, Zhu J. In vivo CT imaging of gold nanoparticle-labeled exosomes in a myocardial infarction mouse model. Ann Transl Med 2021;9(6):504. doi: 10.21037/atm-21-981
Gong et al. Visualizing gold nanoparticle labeled exosomes in vivo

regenerative exosomes to myocardial infarction using cardiac homing peptide. Theranostics 2018;8:1869-78.

47. Tian Y, Li S, Song J, et al. A doxorubicin delivery platform using engineered natural membrane vesicle exosomes for targeted tumor therapy. Biomaterials 2014;35:2383-90.

48. Alvarez-Erviti L, Seow Y, Yin H, et al. Delivery of siRNA to the mouse brain by systemic injection of targeted exosomes. Nat Biotechnol 2011;29:341-5.

49. Wahlgren J, De L Karlson T, Brisslert M, et al. Plasma exosomes can deliver exogenous short interfering RNA to monocytes and lymphocytes. Nucleic Acids Res 2012;40:e130.

50. Kooijmans SA, Stremersch S, Braeckmans K, et al. Electroporation-induced siRNA precipitation obscures the efficiency of siRNA loading into extracellular vesicles. J Control Release 2013;172:229-38.

51. Hu L, Wickline SA, Hood JL. Magnetic resonance imaging of melanoma exosomes in lymph nodes. Magn Reson Med 2015;74:266-71.

(English Language Editor: A. Kassem) 